\title{
Construction of a Hurewicz metric space whose square is not a Hurewicz space
}

by

J. M. O'Farrell (Huntsville, Ala.)

Abstract. In this paper we construct a Hurewicz subset of the reals whose square admits a map onto the irrationals. Martin's Axiom is used in the construction. The method is similar to that used by Sierpiński [1] to construct a Lusin subset of the reals whose square admits a map onto the reals.

1. Terminology and notation. We denote the reals by $R$ and the irrationals by $I$. If $X$ and $Y$ are subsets of $R$, then $X \oplus Y=\{x+y: x \in X, y \in Y\}$. A regular topological space $X$ is a Hurewicz space if for every sequence $G_{1}, G_{2}, G_{3}, \ldots$ of open covers of $X$ there exists a cover $G$ of $X$ such that $G=\bigcup\left\{H_{i}: i<\omega\right\}$ where $H_{i} \subseteq G_{i}$ and $H_{i}$ is finite for each $i<\omega$. A set is a $\gamma$-set if for each open $\omega$-cover, $G$, of $X$, there is a sequence $\left\{G_{n}\right\} \subseteq G$ with $\lim G_{n}=X$. If $\left\{A_{n}: n<\omega\right\}$ is a sequence of subsets of a set $X$, then

$$
\lim A_{n}=\left\{x \in X: \exists n_{0}, \forall n \geqslant n_{0}, x \in A_{n}\right\} .
$$

A family of subsets, $\mathscr{A}$, of a set $X$ is said to be an $\omega$-cover of $X$ if for any finite subset $F$ of $X$, there is an $a \in \mathscr{A}$ with $F \subseteq a$.

2. The irrationals are not a Hurewicz space. The square of the set to be constructed has a natural mapping onto the irrationals. The continuous image of a Hurewicz space must also be a Hurewicz. space, thus the square of the constructed space cannot be a Hurewicz space by the fact that the irrationals are not a Hurewicz space. We describe here an example of a sequence of open covers of the irrationals not witnessing the Hurewicz property. We use the representation $I=N^{N}$ for the irrationals. The sequence of open covers of $N^{N}$ are defined as follows:

$$
G_{n}=\left\{\left(i_{1}, i_{2}, i_{3}, \ldots, i_{n}\right) \times N \times N \times \ldots: i_{j} \in N \text {, for each } j, 1 \leqslant j \leqslant n\right\} .
$$

Notice that this is also a basis for the irrationals not witnessing the Hurewicz. property.

3. Auxillary lemma. In this section we assume Martin's Axiom. Specifically we use the fact that in a locally compact, ccc, Hausdorff space the intersection of $<2^{\infty}$ dense open sets is nonempty. 
3.1. LemMA. Let $S \subseteq I$ with cardinality $|S|=x<2^{\omega}$ such that $S \oplus S \subseteq I, T \subseteq R$ such that $T$ is the complement of the union of $<2^{\omega}$ closed nowhere dense subsets of $R$ and $p \in I$. Then there cxists a point $x \in T \cap I$ such that $(p-x) \in T \cap I$ and $(\{x\} \oplus S) \cup(\{p-x\} \oplus S) \subseteq 1$.

Proof. Define $A_{s}=I \ominus\{s\}=\{i-s: i \in I\}$ for each $s \in S$. Each $A_{s}$ is a dense $G_{\delta}$ set in $R$. We note for any $x \in R, Y_{z} \subseteq R, z \in Z$, the following relation is true:

$$
\{x\} \ominus \cap\left\{Y_{z}: z \in Z\right\}=\bigcap\left\{\{x\} \ominus Y_{z}: z \in Z\right\} .
$$

Therefore for $p \in I$

$$
\{p\} \ominus \cap\left\{A_{s}: s \in S\right\}=\bigcap\left\{\{p\} \ominus A_{s}: s \in S\right\} .
$$

By Martin's Axiom the set

$$
W=\left(\cap\left\{A_{s}: s \in S\right\}\right) \cap\left(\bigcap\left\{\{p\} \ominus A_{s}: s \in S\right\}\right) \cap T \cap I
$$

is nonempty. Let $x \in W$ and $s \in S$. Since $x \in \cap\left\{A_{s}: s \in S\right\}$, for each $s^{\prime} \in S$ there exists an $i_{s^{\prime}} \in I$ such that $x=i_{s^{\prime}}-s^{\prime}$. Therefore $x+s=\left(i_{s}-s\right)+s=i_{s} \in I$. Also if $x \in W$, then $x=p-y$ for some $y \in \cap\left\{A_{s}: s \in S\right\}$, thus $y=p-x$ and $y \in W$. It then follows as before for $s \in S, y+s=(p-x)+s \in I$

4. Construction. Let $\left\{M_{\alpha}: \alpha<2\right\}^{\omega}$ well order the closed uncountable nowhere dense subsets of $R$ and $\left\{y_{\alpha}: \alpha<2^{\omega}\right\}$ well order $I$. Define $S_{1}=\left\{y_{1} / 2\right\}$ and apply the lemma with $S=S_{1}, p=y_{2}, T=R-M_{1}$. We obtain $S_{2}=S_{1} \cup\{x, y-x\}$ where $S_{2}$ has the following properties:
1) $S_{2} \oplus S_{2} \subseteq I$
2) $y_{2} \in S_{2} \oplus S_{2}$
3) $\left(S_{2}-S_{1}\right) \subseteq\left(R-M_{1}\right)$.
1) $S_{\alpha} \oplus S_{\alpha} \subseteq I$,
2) $y_{\alpha} \in S_{\alpha} \oplus S_{\alpha}$
3) For $\delta<\alpha, S_{\delta} \subset S_{\alpha}$
4) Cardinality $\left|S_{\alpha}\right|<2^{\omega}$ and,
5) $\left(S_{\alpha}-\bigcup\left\{S_{\delta}: \delta<\alpha\right\}\right) \subseteq\left(R-\bigcup\left\{M_{\delta}: \delta<\alpha\right\}\right)$.

Assume for each $\alpha<\beta<2^{\omega}$ we have constructed sets $S_{\alpha}$ such that:

Let $S=\bigcup\left\{S_{\alpha}: \alpha<\beta\right\}, p=y_{\beta}, T=\left(R-\bigcup\left\{M_{\alpha}: \alpha<\beta\right\}\right)$ and let $S_{\beta}$ denote the set resulting from application of the lemma. Define $L=\bigcup\left\{S_{\alpha}: \alpha<2^{\omega}\right\}$.

Let $\left\{G_{i}: i<\omega\right\}$ be a sequence of open covers of $L$. Since $L$ is separable, we choose $\left\{x_{i}: i<\omega\right\}$ a countable dense subset of $L$. For each $x_{i}$ choose $g_{i} \in G_{i}$ such that $x_{i} \in g_{i}$. Then $v=\bigcup\left\{g_{i}: i<\omega\right\}$ is dense in $L$ and $(L-v)$ is contained in a closed nowhere dense subset, $M_{\alpha}$, of $R . L \cap M_{\alpha}$ has cardinality $<2^{\omega}$ and hence by Martin's Axiom $L \cap M_{\alpha}$ is a $\gamma$-set [2]. It is known that $\gamma$-sets have the Hurewicz property; in fact, $\gamma$-sets satisfy property $C^{\prime \prime}$. Therefore we may find a sequence $F_{i}$ such that $F_{i} \subseteq G_{i}$, each $F_{i}$ is finite and $\left\{F_{i}: i<\omega\right\}$ covers $L \cap M_{\alpha}$. Then for $F_{i}=\left\{g_{i}\right\} \cup F_{i}$, $\cup\left\{F_{i}: i<\omega\right\}$ is a cover of $L . L$ is therefore a Hurewicz space.
4.1. It is known, assuming Martin's Axiom, that for Hurewicz spaces of cardinality $x<2^{\omega}$, if $X$ is a Lindelöf space then $X^{2}$ is a Hurewicz space. A. Lelek ([3], pp. 216-217) has constructed, using the continuum hypothesis, an example of a Hurewicz space $X$ such that $X^{2}$ is not a Hurewicz space. This example used an uncountable subset of the reals possessing the Lusin property and carrying the half-open interval topology. Lelek had asked whether it was necessary to assume the continuum hypothesis in order to construct an example of a Hurewicz space whose square is not a Hurewicz space. It was also asked in the same paper whether there might exist an example of such a space that was also a metric space. The problem now is whether there is an example of a Hurewicz metric space whose square is not a Hurewicz space, which can be constructed with assumptions weaker than Martin's Axiom.

The author is grateful to the referee for providing modifications which simplified the original proofs yet enhanced the content of the paper.

\section{References}

[1] W. Sierpiński, Sur le produit combinatoire de deux ensembles joissant de la propriété (C), Fund. Math., 24 (1935), 45-50.

[2] F. G alvin and A. W. Miller, $\gamma$-sets and other singular sets of real numbers, Topology Appl. 17 (1984), 145-155.

[3] A. Lelek, On some cover properties of spaces, Fund. Math. 64 (1969), 209-218.

2802 Barcody Circlo

HUNTSVILLE, ALABAMA 3580

Received 17 September 1984;

in revised form 19 August 1985 